\title{
Protective Role of ADA* 2 Allele of the Adenosine Deaminase Gene Against Recurrent Spontaneous Abortions in Iranian Women
}

Authors: Naeimeh Najafi, Ali-Reza Ahmadi, Reza Hajihosseini, Sepideh Khodaverdi, Atefeh Mohammadi

Publication date: 2019/7/1

Journal: Iranian Red Crescent Medical Journal

Background: Adenosine deaminase (ADA) is involved in recurrent spontaneous abortion (RSA) while normal pregnancy is defined by suppressed cell-mediated immunity.

Objectives: This study aimed to determine the connection between single nucleotide polymorphism (SNP) G22A and protection against RSA.

Methods: In this analytical case-control study, the allele frequency of ADA G22A gene polymorphism was determined in 113 participants including 50 women with RSA and 63 healthy pregnant women using RFLP-PCR to determine if there is a statistically significant difference in the frequency of ADA genotypes between the patient group and the control group.

Results: The frequency of ADA2/ADA2 (AA) genotype was significantly different between RSA patients and controls $(P=0.004)$ while no significant differences were identified in the genotype frequency of ADA1/ADA1 $(\mathrm{GG})(\mathrm{OR}=0.678 ; \mathrm{P}=0.228)$ and ADA1/ADA2 $(G A)(O R=0.976 ; P=0.943) . S N P$ G22A in the ADA gene was associated with the protection against RSA. The frequency of ADA alleles in RSA patients was compared between the age groups, and the results indicated that the ADA2 (A) allele was associated with protection against RSA in patients aged 35 years or younger $(\mathrm{P}<0.0001)$.

Conclusions: The findings showed that women carrying the $\mathrm{ADA}^{*} 2$ allele are protected against RSA.

Keywords:

Adenosine Deaminase , Gene, Pregnancy , Recurrent , SingleNucleotide, Spontaneous Abortion, Polymerase Chain Reaction 